Claire Borsenberger, Helmuth Cremer, Denis Joram, Jean-Marie Lozachmeur and Estelle Malavolti 


\title{
Platform competition: market structure and pricing ${ }^{1}$
}

\author{
Claire Borsenberger ${ }^{2}$, Helmuth Cremer $^{3}$, Denis Joram ${ }^{4}$ \\ Jean-Marie Lozachmeur ${ }^{5}$ and Estelle Malavolti ${ }^{6}$
}

April 2019

\footnotetext{
${ }^{1}$ We thank Yassin Lefouilli for his comments and suggestions. ${ }^{2}$ DRAIE, Groupe La Poste

${ }^{3}$ TSE, University of Toulouse Capitole ${ }^{4}$ DRAIE, Groupe La Poste

${ }^{5}$ TSE, CNRS and University of Toulouse Capitole

${ }^{6} \mathrm{TSE}, \mathrm{ENAC}$
} 


\begin{abstract}
We consider an e-commerce sector with two retailers (which may be marketplaces) and two delivery operators. Products are differentiated according to the retailer and the mode of delivery. The representation of product differentiation is inspired by the Anderson, De Palma and Thisse (2002) discrete choice model.

We examine vertical integration of a retailer/ delivery operator pair. Vertical restraints like bundling and/or foreclosure are then considered on top of the integration. Vertical integration in itself eliminates double marginalization, which enhances consumers' welfare. On the other hand, it reduces product variety, and the market power it conveys is likely to reduce profits of the remaining firms. Bundling or foreclosure can be expected to further exacerbate these negative effects.

Our most remarkable result is that vertical integration of a single retailer/operator pair will lead to bundling and foreclosure, and possibly the complete exit of the remaining retailers and operators. This is true even when no explicit bundling or foreclosure is put in place on an a priori basis. Consequently, a competition authority that is concerned with total welfare, should not allow the initial merger.
\end{abstract}

Keywords: E-commerce, delivery operators, vertical integration, bundling, foreclosure JEL Codes: L42, L81, L87. 


\section{Introduction}

The significant development of e-commerce and the emergence of market places has been providing numerous benefits to both retailers and customers. In addition, it has been a boon for delivery operator and allowed postal services to compensate at least in part revenue losses due to declining mail volumes. However, increasing concentration of market power and the worry that it may be extended into adjacent markets has turned into a major concern of policy makers and competition authorities. While there appears to be a wide consensus that "traditional" regulatory or competition policy may have to be amended within the context of platforms, there are so far few rigorous studies that can provide guidance. In this paper we take a step in this direction.

We consider an e-commerce sector with two retailers (which may be marketplaces) and two delivery operators. Products are differentiated according to the retailer and the mode of delivery. Consequently there are four variants of the product. Integration and/or delivery restrictions will reduce product variety; some of the four variants will no longer be available. The representation of product differentiation is inspired by the Anderson, De Palma and Thisse (2002) discrete choice model.

We study several scenarios, starting with a reference case without integration or delivery restrictions. Then, we examine how the equilibrium is affected by the vertical integration of a retailer/ delivery operator pair. Next, vertical restraints like bundling and/or foreclosure are considered on top of the integration. ${ }^{1}$ Vertical integration in itself eliminates double marginalization for the concerned products. This enhances consumers' welfare. On the other hand, it reduces product variety, and the market power it conveys is likely to reduce profits of the remaining firms. Bundling or foreclosure can be expected to further exacerbate these negative effects.

Profits, prices and consumer welfare in these scenarios demonstrate the (anti)competitive effects of integration and strategies like bundling and foreclosure. This, in turn, leads to policy recommendations for competition authorities.

\footnotetext{
${ }^{1}$ In the economic literature, foreclosure is defined as the dominant firm's denial of proper access to an essential good it produces, with the intent of extending monopoly from that segment to an adjacent segment; Rey and Tirole (2007).
} 
The most remarkable result we obtain is that vertical integration of a single retailer/operator pair will lead to bundling and foreclosure and possibly the complete exit of the remaining retailers and operators. This is true even when no explicit bundling or foreclosure is put in place on an a priori basis. The restraints emerge spontaneously as profit maximizing strategies in a Nash equilibrium.

These results lead to the following policy recommendations. When the regulating authority is concerned with total welfare, the initial merger should not be allowed. If, however, a vertical integration has already taken place, competition authorities should also give their clearance to the merger between the two remaining independent entities. This is better for consumers as well as the collectivity as a whole.

\section{The model}

We consider an e-commerce sector with two retailers, which may be marketplaces, and two delivery operators. Products are differentiated according to the retailer and the mode of delivery. Consequently there are four variants of the product. Integration and/or delivery restrictions will reduce product variety; some of the four variants will no longer be available.

Product differentiation is represented by the Anderson-De Palma-Thisse (1992) discrete choice model. This approach is widely used in the Industrial Organization literature to model product differentiation ${ }^{2}$. However, it has not been used to study vertical relationships (integration, bundling or foreclosure).

A differentiated product is sold by downstream sellers $A$ and $B$ (indexed by $j$ ) with marginal cost normalized to 0 and shipped via differentiated upstream postal operators 1 and 2 (indexed by $i$ ) with marginal costs of $c$. Consequently there are potentially four different variants of the product.

There is a mass 1 of consumers. Consumer $l$ derives utility

$$
U_{i j}^{l}=b-p_{i j}+\varepsilon_{i j}^{l}
$$

\footnotetext{
${ }^{2}$ I.e. the process of distinguishing a product or service from others to benefit from consumers heterogeneity of taste.
} 
from consuming good $i j$ where $j=A, B$ and $i=1,2$. The random variables $\varepsilon_{i j}^{l}$ are identically and independently distributed across consumers and products with double exponential distribution over $\mathbb{R}$ with scale parameter $\sigma .^{3}$

The parameter $\sigma$ reflects the degree of product differentiation. When $\sigma$ is small, the different variants are close substitutes and competition is intense. ${ }^{4}$ When $\sigma$ is large, each variant has roughly speaking a local monopoly and competition is not very intense.

Consumers buy their preferred variant of the product if any. Consequently consumer $l$ buys product $i j$ when

$$
U_{i j}^{l} \geq \max _{m n \neq i j}\left\{U_{m n}\right\}
$$

It can be shown that the demand for good $i j$ is then given by

$$
D_{i j}(\mathbf{p})=\frac{\exp \left(-\frac{p_{i j}}{\sigma}\right)}{\sum_{i=1,2} \sum_{j=A, B} \exp \left(-\frac{p_{i j}}{\sigma}\right)}
$$

where $\mathbf{p}=\left(p_{1 A}, p_{1 B}, p_{2 A}, p_{2 B}\right)$ is the vector of consumer prices. Note that the second term in the denominator is absent when there is no outside option. The impact of prices on demand levels are expressed by

$$
\begin{aligned}
\frac{\partial D_{i j}(\mathbf{p})}{\partial p_{i j}} & =\frac{-\frac{1}{\sigma} \exp \left(-\frac{p_{i j}}{\sigma}\right)\left(\sum_{i=1,2} \sum_{j=A, B} \exp \left(-\frac{p_{i j}}{\sigma}\right)\right)+\frac{1}{\sigma} \exp \left(\frac{p_{i j}}{\sigma}\right) \exp \left(-\frac{p_{i j}}{\sigma}\right)}{\left(\sum_{i=1,2} \sum_{j=A, B} \exp \left(-\frac{p_{i j}}{\sigma}\right)\right)^{2}} \\
& =\frac{1}{\sigma}\left(-D_{i j}+D_{i j}^{2}\right) \\
& =\frac{1}{\sigma} D_{i j}\left(D_{i j}-1\right)<0,
\end{aligned}
$$

so that a variant's market share is not surprisingly a decreasing function of its price.

Further we have

$$
\begin{aligned}
\frac{\partial D_{i j}(\mathbf{p})}{\partial p_{m n}} & =\frac{\frac{1}{\sigma} \exp \left(-\frac{p_{i j}}{\sigma}\right) \exp \left(-\frac{p_{m n}}{\sigma}\right)}{\left(\sum_{i=1,2} \sum_{j=A, B} \exp \left(-\frac{p_{i j}}{\sigma}\right)\right)^{2}} \\
& =\frac{1}{\sigma} D_{i j} D_{m n}>0,
\end{aligned}
$$

\footnotetext{
${ }^{3}$ The distribution function of the double exponential distribution is $F(x)=\exp \left(-\exp \left(-\frac{x}{\sigma}\right)\right)$.

${ }^{4}$ When the distribution of $x$ is given by $F(x)=\exp \left(-\exp \left(-\frac{x}{\sigma}\right)\right)$, a smaller $\sigma$ means that there is a larger probability of $x$ exceeding a given threshold. This can be interpreted as the products supplied being closer substitutes.
} 
so that demand for any good increases if the price of one of the other variants increases. This shows that the variants are indeed substitutes and also illustrates the role of the parameter $\sigma$. In particular the cross price effect is the larger the smaller is $\sigma$.

Expected consumer surplus is given by

$$
C S=\sigma \ln \left(\sum_{i=1,2} \sum_{j=A, B} \exp \left(\frac{b-p_{i j}}{\sigma}\right)\right),
$$

see Ben-Akiva and Lerman (1979), p.114.

In the simplest version of this model the market is fully covered. Each consumer buys one of the variants. This is convenient and often used in industrial economics models, but it has the disadvantage that absolute price levels do not matter. Relative prices are important because they affect the allocation of consumers across variants but multiplying all prices by a positive constant has no impact on the outcome (at least as far as total surplus and demand levels are concerned). To introduce adjustments at the extensive margin, we can introduce an outside option as a fifth variant with a given price $p_{0} .{ }^{5}$ Then the levels of prices also matter and welfare measures are more meaningful. The numerical results for both cases are reported separately, the ones without outside option are in appendix.

Note that the outside option can be interpreted as a competitive fringe which sells a homogenous product at marginal cost. From that perspective, we extend the dominant firm model (à la Panzar) from a monopoly to a duopoly (where both upstream and downstream firms behave strategically).

We consider different scenarios with or without integration and/or exclusion or bundling. We state the problem and define the underlying game and specifically its timing. We start with the unrestricted case where retailers and operators are independent and there are no vertical restraints. Then we define the different scenarios.

For the sake of interpretation, note that within this model, integration and vertical

$$
\begin{aligned}
& { }^{5} \text { In that case expression (1) becomes } \\
& \qquad D_{i j}(\mathbf{p})=\frac{\exp \left(-\frac{p_{i j}}{\sigma}\right)}{\sum_{i=1,2} \sum_{j=A, B} \exp \left(-\frac{p_{i j}}{\sigma}\right)+\exp \left(-\frac{p_{0}}{\sigma}\right)}
\end{aligned}
$$

and the subsequent expressions have to be amended in a straighforward way. 
restraints affect prices but also product variety. Restraints will reduce the number of variants available to consumers which tends to decrease welfare - unless of course compensated by a sufficient decrease in prices.

In the symmetric case, the model can be solved analytically but the expressions are not very telling; see Anderson et al. (1992). In the asymmetric cases, obtaining analytical closed form solutions would be at best very tedious. However, the model has essentially only one or two parameters depending on whether we consider an outside option or not. When there is no outside option, the only relevant parameter is $\sigma$. The cost $c$ plays no role and can be normalized to any (strictly positive) level. When there is an outside option, the parameters are $\sigma$ and $p_{0}$. To be more precise, the crucial second parameter is not the absolute level of $p_{0}$ but the ratio $p_{0} / c$.

Consequently numerical solutions are just as informative as analytical expressions and with one or two parameters only, their robustness is easy to verify. Comparing the scenarios tells out how integration, foreclosure or bundling affect profits and thus entry as well as consumer surplus and overall welfare.

\section{The game without integration or restrictions}

We start with a reference scenario with no integration or delivery restrictions, which is represented in Figure 1. Upstream, the delivery operators compete and set their linear delivery rates for each retailer. Retailers compete for the final customers for whom variants of the product are differentiated according to the retailer and the delivery operator. Each retailer can use both delivery operators.

The timing of the game is as follows. In a first stage delivery operators $i=1,2$ simultaneously set rates prices $t_{i A}$ and $t_{i B}$ for retailers $A$ and $B$ respectively. Their profit is given by

$$
\Pi_{i}=\sum_{j}\left(t_{i j}-c\right) D_{i j}(.), i=1,2 .
$$

In stage 2 , retailers $j=A, B$ simultaneously set their prices $p_{1 j}$ and $p_{2 j}$ by taking as 
Postal

Operators

Sellers

Consumers

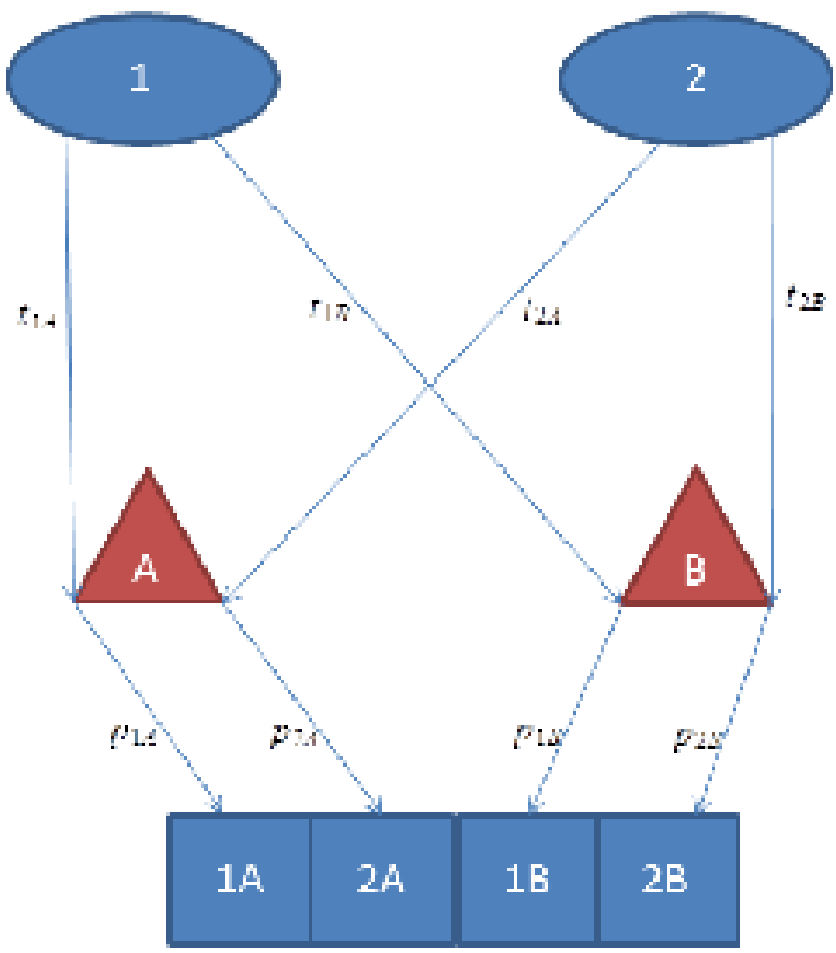


given the delivery rates. Their profit is given by

$$
\pi_{j}=\sum_{i}\left(p_{i j}-t_{i j}\right) D_{i j}(.), j=1,2
$$

Note that a game with the opposite sequence would not be well defined and doesn't make sense. We determine the subgame perfect equilibrium of the game which, as usual, is solved by backward induction. We briefly explain the main steps for this scenario. To avoid repetitions we skip this part for the subsequent scenarios.

\subsection{Stage 2}

The problem of retailer $j$ is given by

$$
\max _{p_{1 j}, p_{2 j}} \pi_{j}=\sum_{i}\left(p_{i j}-t_{i j}\right) D_{i j}(\mathbf{p})
$$

with first order conditions

$$
\begin{aligned}
& D_{1 j}+\left(p_{1 j}-t_{1 j}\right) \frac{\partial D_{1 j}}{p_{1 j}}=0 \\
& D_{2 j}+\left(p_{2 j}-t_{2 j}\right) \frac{\partial D_{2 j}}{p_{2 j}}=0
\end{aligned}
$$

which yields

$$
\begin{aligned}
& 1-\left(p_{1 j}-t_{1 j}\right) \frac{1}{\sigma}\left(1-D_{1 j}(\mathbf{p})\right)=0 \\
& 1-\left(p_{2 j}-t_{2 j}\right) \frac{1}{\sigma}\left(1-D_{2 j}(\mathbf{p})\right)=0 .
\end{aligned}
$$

for $j=A, B$.

This defines the second stage equilibrium prices $\mathbf{p}(\mathbf{t})=\left(p_{1 A}(\mathbf{t}), p_{1 B}(\mathbf{t}), p_{2 A}(\mathbf{t}), p_{2 B}(\mathbf{t})\right)$ as functions of $\mathbf{t}=\left(t_{1 A}, t_{1 B}, t_{2 A}, t_{2 B}\right)$, the vector of delivery rates.

\section{$3.2 \quad$ Stage 1}

In this stage, operators anticipate the equilibrium induced in stage 2. Problem of operator $i=1,2$ is

$$
\max _{t_{i A}, t_{i B}} \Pi_{i}=\sum_{j}\left(t_{i j}-c\right) D_{i j}(\mathbf{p}(\mathbf{t})), i=1,2
$$


with first order condition

$$
\begin{aligned}
& D_{i A}+\left(t_{i A}-c\right) \sum_{l=1,2} \sum_{j=A, B} \frac{\partial D_{i A}}{\partial p_{l j}} \frac{\partial p_{l j}}{\partial t_{i A}}+\left(t_{i B}-c\right) \sum_{l=1,2} \sum_{j=A, B} \frac{\partial D_{i B}}{\partial p_{l j}} \frac{\partial p_{l j}}{\partial t_{i A}}=0 \\
& D_{i B}+\left(t_{i B}-c\right) \sum_{l=1,2} \sum_{j=A, B} \frac{\partial D_{i B}}{\partial p_{l j}} \frac{\partial p_{l j}}{\partial t_{i B}}+\left(t_{i A}-c\right) \sum_{l=1,2} \sum_{j=A, B} \frac{\partial D_{i A}}{\partial p_{l j}} \frac{\partial p_{l j}}{\partial t_{i B}}=0
\end{aligned}
$$

Solving this system of equations for $i=1,2$ yields the vector of equilibrium delivery rates which in turn determine the equilibrium retail prices $\mathbf{p}(\mathbf{t})$ in the first stage.

\section{The game with integration without delivery restrictions}

Assume now that retailer $A$ and operator 1 are integrated. The integrated firm sells and delivers good $A$, the marginal cost of which is $c$. The integrated firm can also deliver good $B$ in quantity $D_{1 B}$ at a rate $t_{1 B}$ and may have good $A$ delivered by firm 2 at rate $t_{2 A}$ and in quantity $D_{2 A}$. This scenario is represented in Figure 2.

The timing of the game is as follow. In stage 1 , the integrated firm chooses $t_{1 B}$ such that it maximizes

$$
\Pi_{1}^{I}=\left(p_{1 A}-c\right) D_{1 A}(\mathbf{p})+\left(p_{2 A}-t_{2 A}\right) D_{2 A}(\mathbf{p})+\left(t_{1 B}-c\right) D_{1 B}(\mathbf{p})
$$

and delivery operator 2 chooses $t_{2 A}$ and $t_{2 B}$ to maximize

$$
\Pi_{2}=\sum_{j}\left(t_{2 j}-c\right) D_{2 j}(\mathbf{p})
$$

In stage 2 , retailers once again simultaneously choose their prices. For firm $B$, the problem is exactly the same as in the previous scenario; it sets prices $p_{1 B}$ and $p_{2 B}$ to maximizes

$$
\pi_{B}=\sum_{i=1,2}\left(p_{i B}-t_{i B}\right) D_{i B}(\mathbf{p})
$$

The problem of the integrated firm, on the other hand is different as it maximizes total profits from its upstream and downstream activities. Formally, it chooses $p_{1 A}$ and $p_{2 A}$ to maximize.

$$
\Pi_{1}^{I}=\left(p_{1 A}-c\right) D_{1 A}(\mathbf{p})+\left(p_{2 A}-t_{2 A}\right) D_{2 A}(\mathbf{p})+\left(t_{1 B}-c\right) D_{1 B}(\mathbf{p}) .
$$


Postal

Operators

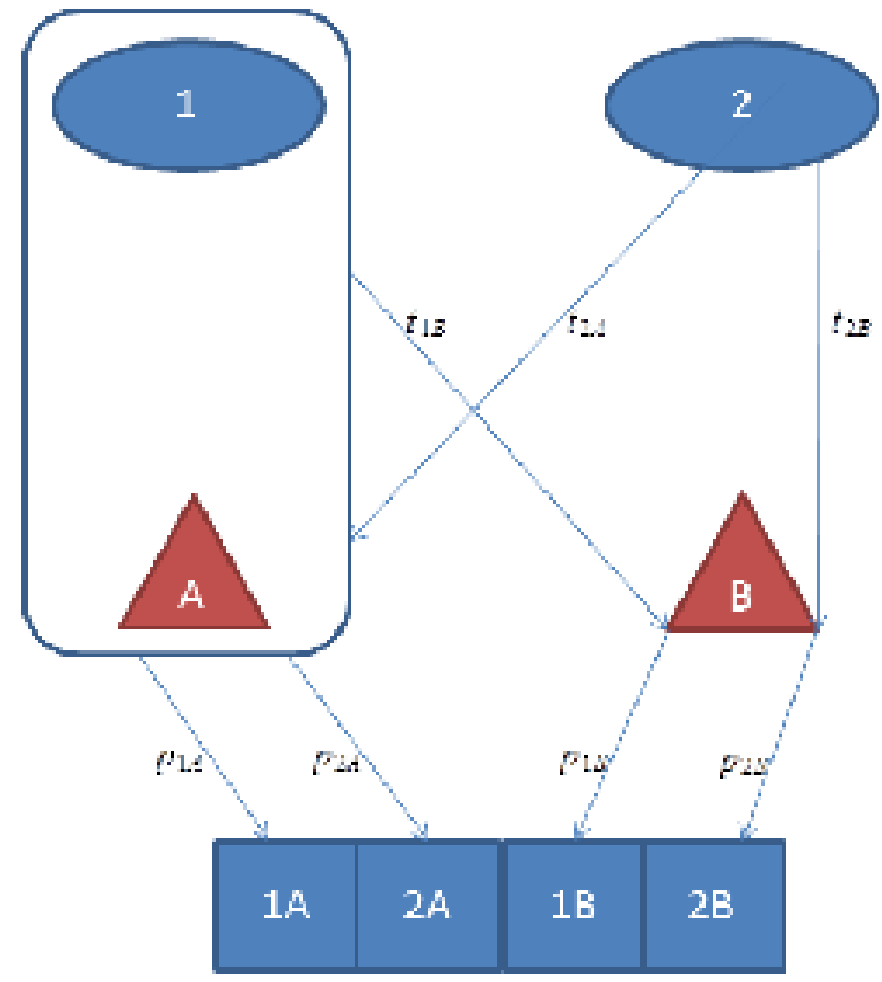




\section{The game with integration and bundling}

Compared to the previous scenario, we add the extra constraint that there is bundling in the sense that the product sold by firm $A$ must be delivered by the integrated operator 1. Variant $2 A$ of the product thus no longer exists. Let $\mathbf{p}^{F A}=\left(p_{A}, p_{1 B}, p_{2 B}\right)$ the prices of the remaining variants. The demand functions for these variants follow directly from equation (1). ${ }^{6}$ This scenario is represented in Figure 3.

The timing of the game is as follow. In Stage 1, the integrated firm chooses $t_{1 B}$ to maximize

$$
\Pi_{1}^{I}=\left(p_{A}-c\right) D_{A}\left(\mathbf{p}^{F A}\right)+\left(t_{1 B}-c\right) D_{1 B}\left(\mathbf{p}^{F A}\right),
$$

while delivery operator 2 chooses $t_{2 B}$ to maximize

$$
\Pi_{2}=\left(t_{2 B}-c\right) D_{2 B}\left(\mathbf{p}^{F A}\right) .
$$

In Stage 2, the integrated firm sets $p_{A}$ in order to maximize

$$
\Pi_{1}^{I}=\left(p_{A}-c\right) D_{A}\left(\mathbf{p}^{F A}\right)+\left(t_{1 B}-c\right) D_{1 B}\left(\mathbf{p}^{F A}\right),
$$

while retailer $B$ sets its prices $p_{1 B}$ and $p_{2 B}$ to maximize

$$
\pi_{B}=\sum_{i=1,2}\left(p_{i B}-t_{i B}\right) D_{i B}(\mathbf{p})
$$

\section{The game with integration and foreclosure}

Assume now that the delivery operator which is part of the integrated firm does not deliver good $B$. This yields the scenario depicted in Figure 4. Again, one variety disappears namely $1 B$ and we define $\mathbf{p}^{F B}=\left(p_{A 1}, p_{A 2}, p_{B}\right)$ as the vector of prices of the

${ }^{6}$ We have for $i=1,2$.

$$
\begin{aligned}
D_{A}\left(\mathbf{p}^{F A}\right) & =\frac{\exp \left(-\frac{p_{A}}{\sigma}\right)}{\exp \left(-\frac{p_{A}}{\sigma}\right)+\sum_{i=1,2} \exp \left(-\frac{p_{i B}}{\sigma}\right)}, \\
D_{i B}\left(\mathbf{p}^{F A}\right) & =\frac{\exp \left(-\frac{p_{i B}}{\sigma}\right)}{\exp \left(-\frac{p_{A}}{\sigma}\right)+\sum_{i=1,2} \exp \left(-\frac{p_{i B}}{\sigma}\right)} .
\end{aligned}
$$


Postal

Operators

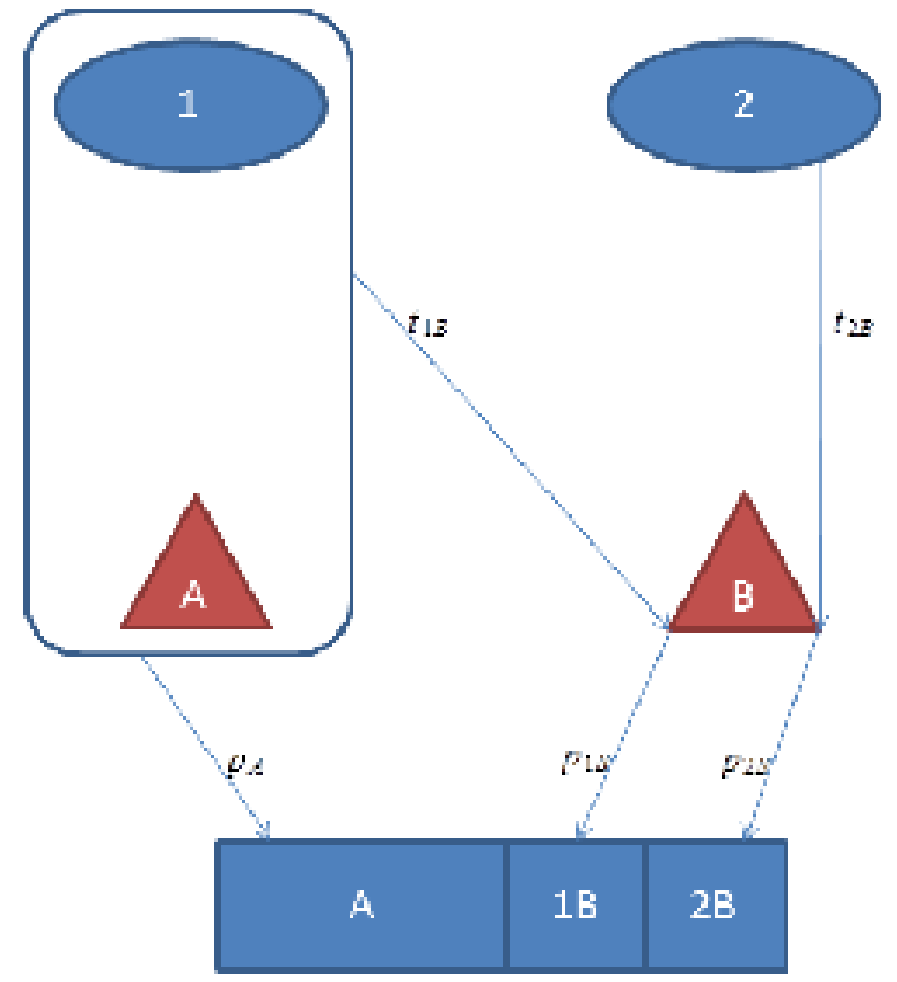




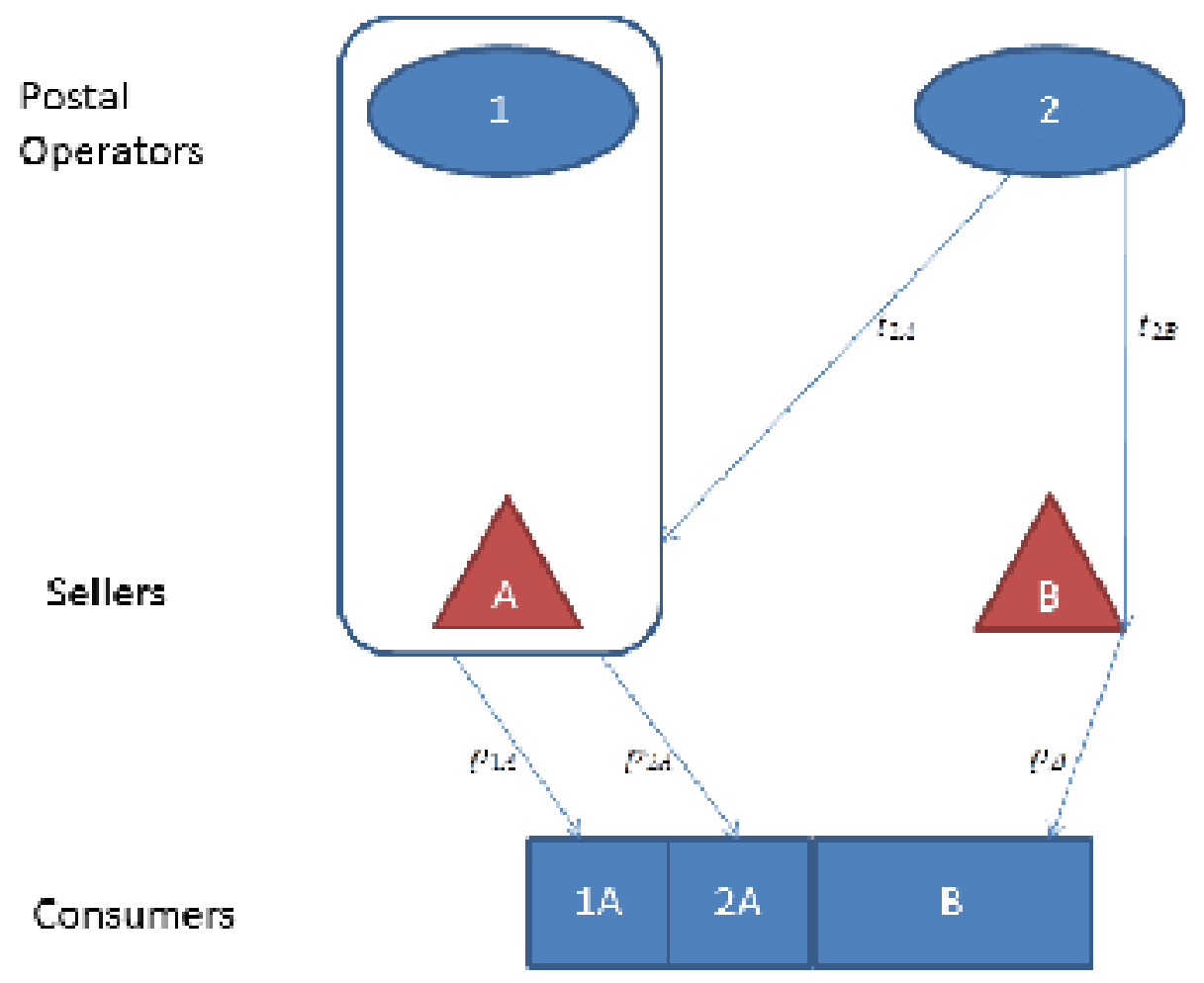

remaining variants. The derivation of the demand functions $D_{i A}\left(\mathbf{p}^{F A}\right)$ and $D_{B}\left(\mathbf{p}^{F A}\right)$ is straightforward. ${ }^{7}$

The timing of the game is as follow. In the first stage, delivery operator 2 is the sole

${ }^{7}$ We have

$$
\begin{aligned}
D_{i A}\left(\mathbf{p}^{F A}\right) & =\frac{\exp \left(-\frac{p_{i A}}{\sigma}\right)}{\exp \left(-\frac{p_{B}}{\sigma}\right)+\sum_{i=1,2} \exp \left(-\frac{p_{i A}}{\sigma}\right)} \\
D_{B}\left(\mathbf{p}^{F A}\right) & =\frac{\exp \left(-\frac{p_{B}}{\sigma}\right)}{\exp \left(-\frac{p_{B}}{\sigma}\right)+\sum_{i=1,2} \exp \left(-\frac{p_{i A}}{\sigma}\right)}
\end{aligned}
$$


active player and chooses $t_{2 A}$ and $t_{2}$ to maximize

$$
\Pi_{2}=\left(t_{2 A}-c\right) D_{2 A}\left(\mathbf{p}^{F B}\right)+\left(t_{2 B}-c\right) D_{B}\left(\mathbf{p}^{F B}\right) .
$$

In Stage 2, retailer $B$ chooses its price $p_{B}$ like in the previous scenarios, that is to maximize

$$
\pi_{B}=\left(p_{B}-t_{2 B}\right) D_{B}\left(\mathbf{p}^{F B}\right)
$$

The integrated firm simultaneously sets $p_{1 A}$ and $p_{2 A}$ and to maximize the sum of upstream and downstream profits given by

$$
\Pi_{1}^{I}=\left(p_{1 A}-c\right) D_{1 A}\left(\mathbf{p}^{F B}\right)+\left(p_{2 A}-t_{2 A}\right) D_{2 A}\left(\mathbf{p}^{F B}\right) .
$$

\section{The game with integration and total bundling/foreclosure}

Assume now that the integrated retailer delivers good $A$ only via its own operator and that the integrated delivery operator does not deliver good $B$; see Figure 5 . We are now left with two variants with prices $\mathbf{p}^{F}=\left(p_{A}, p_{B}\right){ }^{8}$

The timing follows the same logic as in the previous scenarios. In the first stage, delivery operator 2 is the only active player and chooses $t_{2}$ to maximize

$$
\Pi_{2}=\left(t_{2}-c\right) D_{A}\left(\mathbf{p}^{F}\right)
$$

In stage 2 , the integrated firm chooses $p_{A}$ to maximize

$$
\Pi_{1}^{I}=\left(p_{A}-c\right) D_{A}\left(\mathbf{p}^{F}\right)
$$

while the problem of retailer $B$ continues to be the same as in the previous scenarios: it simultaneously chooses its price $p_{B}$ to maximize

$$
\pi_{B}=\left(p_{B}-t_{2}\right) D_{B}\left(\mathbf{p}^{F}\right)
$$

${ }^{8}$ Demand functions are given by

$$
\begin{aligned}
& D_{A}\left(\mathbf{p}^{F}\right)=\frac{\exp \left(-\frac{p_{A}}{\sigma}\right)}{\exp \left(-\frac{p_{A}}{\sigma}\right)+\exp \left(-\frac{p_{B}}{\sigma}\right)}, \\
& D_{B}\left(\mathbf{p}^{F}\right)=\frac{\exp \left(-\frac{p_{B}}{\sigma}\right)}{\exp \left(-\frac{p_{A}}{\sigma}\right)+\exp \left(-\frac{p_{B}}{\sigma}\right)} .
\end{aligned}
$$


Postal

Operators

Consumers

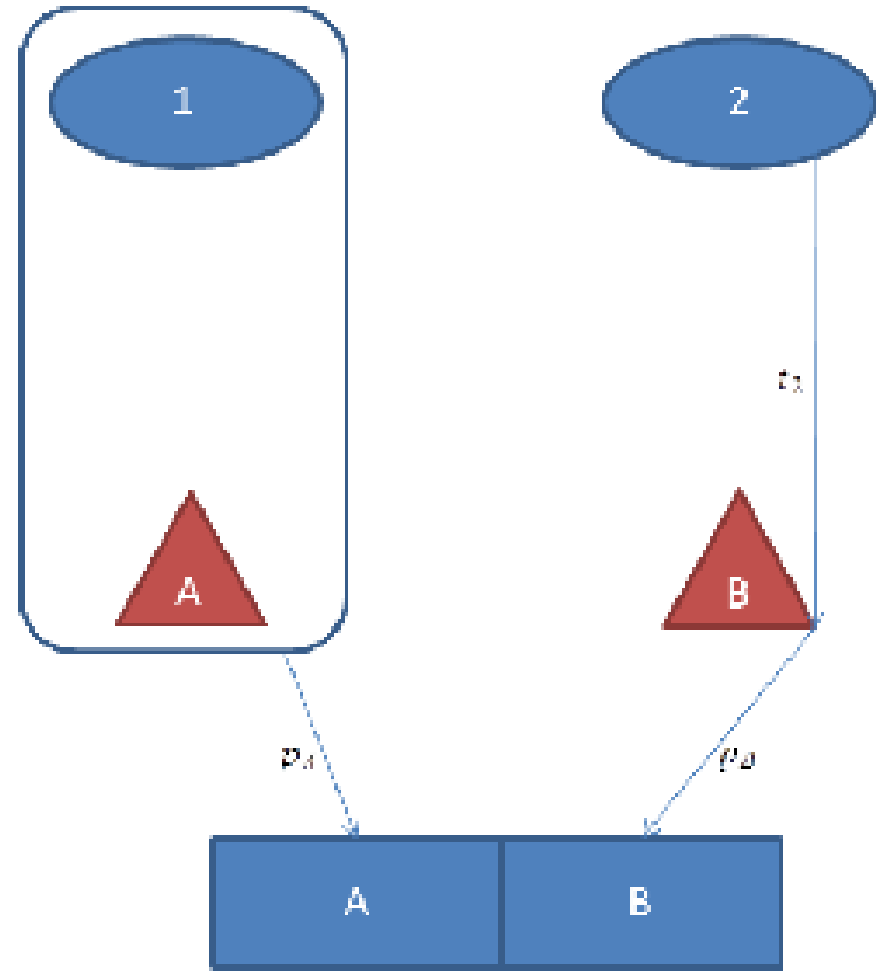




\section{Double integration}

Finally, we consider the case where both retailer-operator pairs integrate: retailer $A$ with operator 1 and retailer $B$ with operator 2 . This scenario is not directly relevant for studying the issues of bundling and foreclosure. However, it is interesting to draw a complete picture of the implications of vertical integration. Furthermore this scenario is useful for comparing the results obtained in this setting with product differentiation to those obtained in a homogenous product model by Borsenberger et al. (2018).

We skip the formal definition of this game which follows in a straightforward way from the previous scenarios. Like in Section 7 there are only two variants left and demand functions are as specified in footnote 8 . Roughly speaking the game now reduces to a single stage, where the integrated firms choose $p_{1 A}$ and $p_{2 B}$ to maximize their respective profits and we calculate the Nash equilibrium of this game.

\section{Numerical results}

The equilibria in the various scenarios are reported in Tables $1-4$. The tables for the most are self-explanatory. The first column is the equilibrium without integration. The second represents integration without restrictions. The third is integration plus bundling, the fourth integration plus foreclosure, the fifth integration with foreclosure and bundling - all as defined above. The last column is the double integration scenario. For each scenario we report all the relevant equilibrium prices, delivery rates, profits of retailers and delivery operators, demand levels as well as consumer $(C S)$ and total surplus (TS). The symbol - in a cell means that the corresponding variable is not relevant. The symbol $*$ (for prices or delivery rates) means that there is a "corner solution": demand drops to zero (the price is then not uniquely determined - all levels sufficiently large to yield zero demand are equivalent). In all simulations $c$ is set to 1 ; this is essentially just a normalization.

The simulations have shown that results are robust and that two main cases have to be considered: low $\sigma$-variants are relatively close substitutes on the one hand and large $\sigma$-variants are not as easily substitutable on the other hand. While the overall 


\begin{tabular}{|l|c|c|c|c|c|c|}
\hline & No Int. & Int. w/ restriction & Int. bundling & Int. foreclosure & Int. for. \& b. & Double Int. \\
\hline$p_{1 A}$ & 2.92 & 2.60 & 2.86 & 2.60 & 2.60 & 1.94 \\
\hline$p_{2 A}$ & 2.92 & $*$ & - & $*$ & - & - \\
\hline$p_{1 B}$ & 2.92 & $*$ & 2.63 & $*$ & - & - \\
\hline$p_{2 B}$ & 2.92 & $*$ & 2.94 & - & $*$ & 1.94 \\
\hline$t_{1 A}$ & 2.08 & - & - & - & - & - \\
\hline$t_{2 A}$ & 2.08 & $*$ & - & $*$ & - & - \\
\hline$t_{1 B}$ & 2.08 & $*$ & 1.61 & - & - & - \\
\hline$t_{2 B}$ & 2.08 & $*$ & 1.98 & $*$ & $*$ & - \\
\hline$\pi_{1}$ & 0.44 & 1.10 & 0.67 & 1.10 & 1.10 & 0.44 \\
\hline$\pi_{2}$ & 0.44 & 0 & 0.19 & 0 & 0 & 0.44 \\
\hline$\pi_{A}$ & 0.34 & - & - & - & - & - \\
\hline$\pi_{B}$ & 0.34 & 0 & 0.58 & 0 & 0 & - \\
\hline$D_{1 A}$ & 0.20 & 0.68 & 0.23 & 0.68 & 0.68 & 0.47 \\
\hline$D_{2 A}$ & 0.20 & 0 & - & 0 & - & - \\
\hline$D_{1 B}$ & 0.20 & 0 & 0.37 & - & - & - \\
\hline$D_{2 B}$ & 0.20 & 0 & 0.20 & 0 & 0 & 0.47 \\
\hline$C S$ & 7.86 & 7.58 & 7.85 & 7.58 & 7.58 & 8.43 \\
\hline$T S$ & 9.44 & 8.68 & 9.30 & 8.68 & 8.68 & 9.32 \\
\hline
\end{tabular}

Table 1: Outside option such that $p_{0}=3 c$; low $\sigma=0.5$.

picture is the same, some specific results differ according to whether an outside option is available or not. We concentrate on the cases where an outside option is available because welfare measures are more meaningful in this case. Table 1 presents the results with a low $\sigma(\sigma=0.5)$ while Table 2 considers a larger level of $\sigma(\sigma=1)$. The counterparts to these results for the case without outside option are presented in the appendix. Within each of the two cases that emerge, different levels of $\sigma$ and $p_{0}$ appear to yield the same pattern of results. 


\begin{tabular}{|l|c|c|c|c|c|c|}
\hline & No Int. & Int. w/ restriction & Int. bundling & Int. foreclosure & Int. for. \& b. & Double Int. \\
\hline$p_{1 A}$ & 8.31 & 5.65 & 8.06 & 5.98 & 5.65 & 4.68 \\
\hline$p_{2 A}$ & 8.31 & $*$ & - & 8.44 & - & - \\
\hline$p_{1 B}$ & 8.31 & $*$ & 7.12 & 7.36 & - & - \\
\hline$p_{2 B}$ & 8.31 & 7.27 & 8.38 & - & 7.27 & 4.68 \\
\hline$t_{1 A}$ & 5.05 & - & - & - & - & - \\
\hline$t_{2 A}$ & 5.05 & $*$ & - & 3.45 & - & - \\
\hline$t_{1 B}$ & 5.05 & $*$ & 3.23 & - & - & - \\
\hline$t_{2 B}$ & 5.05 & 4.59 & 4.68 & 4.76 & 4.59 & - \\
\hline$\pi_{1}$ & 1.56 & 2.65 & 2.37 & 2.98 & 2.65 & 1.68 \\
\hline$\pi_{2}$ & 1.56 & 0.91 & 0.69 & 1.20 & 0.91 & 1.68 \\
\hline$\pi_{A}$ & 1.26 & - & - & - & - & - \\
\hline$\pi_{B}$ & 1.26 & 0.67 & 2.09 & 0.60 & 0.67 & - \\
\hline$D_{1 A}$ & 0.19 & 0.57 & 0.22 & 0.46 & 0.57 & 0.45 \\
\hline$D_{2 A}$ & 0.19 & 0 & - & 0.13 & - & 0.45 \\
\hline$D_{1 B}$ & 0.19 & 0 & 0.35 & - & - & - \\
\hline$D_{2 B}$ & 0.19 & 0.25 & 0.18 & 0.23 & 0.25 & - \\
\hline$C S$ & 4.97 & 5.47 & 4.93 & 5.55 & 5.47 & 6.88 \\
\hline$T S$ & 10.63 & 9.71 & 10.09 & 10.34 & 9.71 & 10.24 \\
\hline
\end{tabular}

Table 2: Outside option with $p_{0}=8 c$; large $\sigma=1$. 
The first remarkable property is that when retailer $A$ integrates, foreclosure and bundling appear "spontaneously" (in equilibrium) even when they are not imposed $e x$ ante. In other words, even when the retailer does not commit to such a policy (say in stage 0), as in the following scenarios, the relevant markets disappear in the Nash equilibrium. Consequently columns 2 and 5 are essentially identical. In particular, the consumer surplus is identical: such spontaneous vertical restraints are not detrimental to consumers. They cannot be considered and condemned as anticompetitive practices when in a first step, merger between upstream and downstream entities has been authorized.

Intuitively, the integrated firm increases the delivery rate applied to the other retailer because this increases the competitor's cost. This increase is so significant that the other retailer no longer uses this delivery option so that we effectively have foreclosure in equilibrium. Note that since variety $1 B$ is no longer available the integrated operator foregoes some revenues but it also reduces competition in the downstream market and this effect dominates. As to the bundling, the integrated firm prefers using its own delivery operator which is cheaper. Once again it foregoes a variety in the process (namely $2 A$ ) but the cost effect dominates.

When $\sigma$ is small so that the products are relatively close substitutes, these forces are so significant that when $A$ and 1 integrate, the remaining retailer and delivery operator exit the market in equilibrium (their demands drop to zero). As the last column shows, they can, however, avoid this outcome by forming their own vertical chain (which would realize a positive profit of 0.44 ).

When $\sigma$ is sufficiently large, on the other hand, retailer $B$ will be able to preserve a positive market share (and profit) in equilibrium and use operator 2's delivery services. Intuitively, products are then sufficiently differentiated to that retailer $A$ cannot capture the entire market. Still integration leads to an equilibrium with both foreclosure and bundling ( $D_{1 B}$ and $D_{2 A}$ drop to zero). Furthermore, like for smaller levels of $\sigma$ it remains true that $B$ and 2 would be better off by forming their own vertical chain.

So far we have considered the cases where the integrated firm does not a priori commit to any vertical restraint. When it commits to both policies, we get exactly the 
same outcome. With a low $\sigma$ this is also true when it only commits to foreclosure. Interestingly, however, when it commits to bundling only, there will be no foreclosure in equilibrium and the independent actors remain in the market, irrespective of the level of $\sigma$. To understand these results, consider first the case where the integrated firm commits to foreclosure. In other words, it foregoes some delivery activity, namely that of the good sold by retailer $B$. In this case, it is in its interest to bundle its own product with delivery by its parcel delivery business unit in order to maximize its delivery activity. This is achieved because under bundling consumers of good $A$ no longer have the option of receiving delivery by operator 2 . Next consider the case where the integrated firms commits to bundle its good and delivery service. In this case, it is not in its interest to practice foreclosure. Quite the opposite; it maximizes the activity of its retailing division by allowing consumers to be delivered by the independent delivery operator. This is reminiscent of a result obtained by Reisinger and Tarantino (2015) who show that when an upstream monopolist integrates with an (inefficient) retailer, the monopolist would prefer maintaining the distribution of its goods through the other retailer. We do not consider differences in retailers' efficiency but like in their setting we have an output-shifting effect as the efficient retailer helps him expanding the output on the final market.

Furthermore, when the integration is associated with commitment to bundling only the remaining firms no longer gain by forming a second vertical chain.

Turning to welfare, our results show that integration of any kind, with or without extra restraints, decreases welfare. Consumer surplus, on the other hand, is highest in the double integration scenario. This is because the absence of double marginalization leads to a drastic decrease in prices which more than outweighs the reduction of product differentiation. However, the increase in consumer surplus is not sufficiently significant to compensate the decrease in producer surplus.

To sum up, vertical integration of a single retailer/operator pair will lead to bundling and foreclosure and possibly the complete exit of the remaining retailers and operators. This is true even when no explicit bundling or foreclosure is put in place on an a priori basis. The restraints emerge spontaneously as profit maximizing strategies in a Nash 
equilibrium.

\section{Concluding comments}

From a competition policy perspective this is an example of a situation where market power in one market segment may spill over to others. This is in line with the results of Rey and Tirole (2007) who show that the anti-competitive effects of exclusionary practices are more important if the market power is in the downstream market. This is because the exclusive practices enhance its monopoly power as it extracts all the profits from the upstream firms and charges monopoly price to final consumers. The treatment of these restraints, initially based on the block exemption Regulation of 1999, is now essentially relying on Regulation 330/2010 and some more recent guidelines. The main evolution, following the growth of massive retailers in Europe and the rise of internet distribution, has been to move toward a more "effect-based" approach. This means that the crucial issue is to determine whether a vertical agreement (or part of it), has actual or potential anti-competitive effects that are not outweighed by pro-competitive effects (or objective justifications); see Petit and Henry (2010).

When the negative effects of the exclusionary practice outweigh the positive effects, some remedies are recommended to restore an outcome that enhances social welfare. The remedies can be either structural ones like the divestiture of the property rights or the prohibition of the vertical merger. There could be behavioral remedies that would consist in imposing unbundling or non discriminatory practices.

In the case we studied, the following policy recommendations could be made: based on the maximization of total welfare criteria, the initial merger should not be allowed. If, however, a vertical integration has already taken place, competition authorities should also give their clearance to the merger between the two remaining independent entities. This is better for consumers as well as the collectivity as a whole. 


\section{References}

[1] Anderson, S., A De Palma and J.F. Thisse, "Discrete Choice Theory of Product Differentiation", MIT Press, 1992.

[2] Ben-Akiva, M. and S. Lerman, "Disaggregate Travel and Mobility Choice Models and Measures of Accessibility." In D.A. Hensher and P.R. Stopher (eds.), Behavioral Travel Modelling, London: Croon Helm, 654-679, 1979.

[3] Borsenberger, C., H. Cremer, D. Joram and J.-M. Lozachmeur, "Vertical integration in the e-commerce sector," in: Parcu, P., T. Brennan and V. Glass, eds, New business and Regulatory Strategies in the Postal Sector, 2018.

[4] EU Guidelines on Vertical Restraints, 2010.

[5] Petit, N. and D. Henry, 2010, "Vertical Restraints under EU Competition Law: Conceptual Foundations and Practical Framework", http://dx.doi.org/10.2139/ssrn.1724891

[6] Reisinger, M. and Tarantino, 2015, "Vertical integration, foreclosure, and productive efficiency", The Rand Journal of Economics, Vol. 46, No. 3 (Fall 2015), pp. 461-479.

[7] Rey, P. and J. Tirole, 2007, "A primer in foreclosure", Chapter 33 in Handbook of Industrial Organization, vol. 3, pp 2145-2220. 


\begin{tabular}{|l|c|c|c|c|c|c|}
\hline & No Int. & Int. w restriction & Int. bundling & Int. foreclosure & Int. for. \& b. & Double Int \\
\hline$p_{1 A}$ & 3.5 & 2.48 & 3.19 & 2.59 & 2.48 & 2 \\
\hline$p_{2 A}$ & 3.5 & $*$ & - & 3.16 & - & - \\
\hline$p_{1 B}$ & 3.5 & $*$ & 2.91 & 2.84 & - & - \\
\hline$p_{2 B}$ & 3.5 & 2.82 & 3.44 & - & 2.82 & 2 \\
\hline$t_{1 A}$ & 2.5 & - & - & - & - & - \\
\hline$t_{2 A}$ & 2.5 & $*$ & - & 1.57 & - & - \\
\hline$t_{1 B}$ & 2.5 & $*$ & 1.67 & - & - & - \\
\hline$t_{2 B}$ & 2.5 & 2.06 & 2.38 & 2.11 & 2.06 & - \\
\hline$\pi_{1}$ & 0.75 & 0.98 & 1.00 & 1.09 & 0.98 & 0.5 \\
\hline$\pi_{2}$ & 0.75 & 0.35 & 0.25 & 0.44 & 0.35 & 0.5 \\
\hline$\pi_{A}$ & 0.5 & - & - & - & - & - \\
\hline$\pi_{B}$ & 0.5 & 0.25 & 0.84 & 0.22 & 0.25 & - \\
\hline$D_{1 A}$ & 0.25 & 0.66 & 0.30 & 0.52 & 0.66 & 0.5 \\
\hline$D_{2 A}$ & 0.25 & 0 & - & 0.16 & - & - \\
\hline$D_{1 B}$ & 0.25 & 0 & 0.52 & 0.32 & - & - \\
\hline$D_{2 B}$ & 0.25 & 0.34 & 0.18 & - & 0.34 & 0.5 \\
\hline$C S$ & 7.19 & 7.72 & 7.40 & 7.73 & 7.72 & 8.34 \\
\hline$T S$ & 9.69 & 9.31 & 9.50 & 9.50 & 9.31 & 9.34 \\
\hline
\end{tabular}

Table 3: No outside option; low sigma $\sigma=0.5$.

\section{Appendix}

\section{A.1 Results without outside option}




\begin{tabular}{|l|c|c|c|c|c|c|}
\hline & No Int. & Int. w/ restriction & Int. bundling & Int. foreclosure & Int. for. \& b. & Double int. \\
\hline$p_{1 A}$ & 11 & 6.93 & 9.79 & 7.36 & 6.93 & 5 \\
\hline$p_{2 A}$ & 11 & $*$ & - & 9.67 & - & - \\
\hline$p_{1 B}$ & 11 & $*$ & 8.66 & 8.37 & - & - \\
\hline$p_{2 B}$ & 11 & 8.28 & 10.78 & - & 8.28 & 5 \\
\hline$t_{1 A}$ & 7 & - & - & - & - & - \\
\hline$t_{2 A}$ & 7 & $*$ & - & 3.31 & - & - \\
\hline$t_{1 B}$ & 7 & $*$ & 3.68 & - & - & - \\
\hline$t_{2 B}$ & 7 & 5.26 & 6.53 & 5.45 & 5.26 & - \\
\hline$\pi_{1}$ & 3 & 3.93 & 4.01 & 4.36 & 3.93 & 2 \\
\hline$\pi_{2}$ & 3 & 1.43 & 1.00 & 1.78 & 1.43 & 2 \\
\hline$\pi_{A}$ & 2 & - & - & - & - & - \\
\hline$\pi_{B}$ & 2 & 1.01 & 3.37 & 0.91 & 1.01 & - \\
\hline$D_{1 A}$ & 0.25 & 0.66 & 0.30 & 0.52 & 0.66 & 0.5 \\
\hline$D_{2 A}$ & 0.25 & 0 & - & 0.16 & - & - \\
\hline$D_{1 B}$ & 0.25 & 0 & 0.52 & 0.32 & - & - \\
\hline$D_{2 B}$ & 0.25 & 0.34 & 0.18 & - & 0.34 & 0.5 \\
\hline$C S$ & 1.77 & 3.89 & 2.63 & 3.94 & 3.89 & 6.38 \\
\hline$T S$ & 11.77 & 10.27 & 11.01 & 11.00 & 10.27 & 10.38 \\
\hline
\end{tabular}

Table 4: No outside option; large $\sigma=1$. 